\title{
LINGUA SPAGNOLA E ITALIANA A CONFRONTO NELLE ANTOLOGIE POETICHE DEL SECONDO CINQUECENTO. NOTE SU ALCUNI SONETTI IN CASTIGLIANO DI AUTORI ITALIANI NEL TEMPIO PER GIOVANNA D'ARAGONA (1555) E NELLE RIME ET VERSI PER GIOVANNA CASTRIOTA CARAFA (1585)
}

\author{
Matteo Lefèvre \\ Universidad de Tor Vergata (Roma)
}

\begin{abstract}
In this essay we analyse through a historical and lexical approach an important problem of Spanish and Italian Renaissance history of poetry and language. Since the second part of the Sixteenth century, in some poetical anthologies published in Italy, together with the "classical" «sonetti» and «canzoni», we find some poems «a la manera italiana» written in Spanish language. The authors of these lyrics are, most of all, Italian poets, who show a deep acknowledgement of Castilian language, certainly due to the daily relations that they had with Spanish literates, captains and ambassadors living in Italy because of the needs of the Imperial administration and army. These Italian poets also disguise themselves and change their names in hispanic forms, in order to look like "perfect" Spanish courtiers. These verses written in Castilian not only represent one orthodox attempt to celebrate, directly in their own language, the most important personalities of Spanish power in Italy, but also show the deep cultural, political and linguistic consciousness of Italian Renaissance poetry.
\end{abstract}

Nella seconda metà del Cinquecento i poeti spagnoli in Italia attivano, nei principali centri della penisola, un circuito linguistico, oltre che letterario, che risponde in pieno all'indirizzo del petrarchismo. I vari gentilhombres che popolano le cancellerie e gli uffici diplomatici di Carlo V e Filippo II in Italia comunicano con l'intelighenzia dei vari stati italiani secondo quelli che sono gli istituti riconosciuti della civiltà rinascimentale, in cui la scrittura poetica - intesa soprattutto come raffinato "gioco di società" e come privilegiato medium encomiastico - occupa un posto fondamentale. Il petrarchismo diviene pertanto il codice di riferimento di questa pratica sociale tanto per i rimatori italiani quanto per quelli spagnoli, e così le diverse miscellanee poetiche che le tipografie veneziane o quelle del Viceregno partenopeo immettono sul mercato nella seconda parte del secolo cominciano a popolarsi di autori non solo italiani ma anche spagnoli, spesso membri dell'aristocrazia o soldati e comandanti degli eserciti imperiali. I poeti spagnoli del Cinquecento arricchirono pertanto forme e contenuti della propria tradizione lirica attraverso la creazione di una nuova lingua poetica di diretta ispirazione petrarchesca, una lingua che obbedisse al principio dell'imitatio styli e che si adeguasse con flessibilità alle strutture piane della versificazione endecasillabica; e quelli, numerosi, che vissero in Italia nel periodo dell'occupazione, arrivarono a possedere una tale padronanza degli strumenti della lirica da divenire poeti bilingue e comporre sonetti e canzoni sia in castigliano che in italiano, 
affollando sempre di più le antologie che si andarono moltiplicando soprattutto negli ultimi decenni del secolo. Ad ogni modo, viceversa, non mancarono neppure poeti italiani che si cimentarono nella composizione di versi nella «universal castellana lengua»: in ossequio alla nazione occupante, o meglio ai suoi rappresentanti in Italia, presso cui spesso lavorarono prestando i servizi più disparati, questi letterati non vollero perdere l'occasione di dimostrare la propria dimestichezza con la lingua dei dominatori, componendo varie liriche in castigliano e inserendole nelle raccolte dedicate a celebrare o ricordare la nobiltà e l'eccellenza di capitani e nobildonne di origine ispanica. È ciò che avviene, come vedremo, nel Tempio per Giovanna d'Aragona $(1555)^{1}$, una delle miscellanee encomiastiche più famose del secondo Cinquecento, che viene pubblicata poco dopo la metà del secolo da Plinio Pietrasanta con la prestigiosa supervisione di Girolamo Ruscelli, letterato sempre pronto a fiutare le opportunità commerciali offerte dalla forma «antologia»: è proprio nell'ultima parte di questa raccolta che compare una sezione di «rime spagnuole», consacrate alla «divina» Giovanna tanto da letterati iberici quanto, soprattutto, da un ferrato manipolo di poeti italiani. Inoltre, quello della raccolta ruscelliana non è un fenomeno isolato: anche nelle successive Rime et versi in onore di Giovanna Castriota Carafa $(1585)^{2}$ è possibile individuare una sezione sufficientemente ampia che raccoglie in serie varie liriche in castigliano, redatte da autori spagnoli e italiani e tutte destinate, comunque, in ossequio alla finalità principale dell'opera, a omaggiare la nobildonna campana. Sul piano storico ed editoriale, prima ancora che linguistico, episodi di questo tipo si moltiplicano durante il secolo per via del consolidamento via via più stabile del dominio e dell'influenza ispanica sulla penisola nonché per la forza propulsiva dell'industria tipografica, tuttavia sarebbe un errore ridurre il peso e l'interesse di queste liriche, composte in lingua spagnola in prevalenza da poeti italiani, alla semplice dimensione dell'elogio e dell'opportunità "diplomatica" o commerciale: di fatto, i molti petrarchisti italiani che accettano di cimentarsi in una lingua non materna testimoniano più che altro della dimensione internazionale del petrarchismo. Ci troviamo di fronte a un movimento poetico che dalla "centrale" italiana non solo impone e diffonde forme e contenuti in tutta Europa, offrendo altresì tipologicamente una vasta gamma di temi e di possibilità di apprendimento ed espressione poetica a tutti i sudditi dell'Impero (e non), ma applica in concreto anche un principio di versatilità linguistica che rende il petrarchismo stesso una sorta di elegante codice sovranazionale, i cui statuti permettono a poeti di nazioni e tradizioni differenti di intendersi e di essere accettati ovunque all'interno dell'alta società cortigiana e cittadina.

Grazie al «gioco sociale» del petrarchismo letterati di paesi in guerra tra loro comunicano su un piano assolutamente pacifico, riconoscendosi nelle strutture della lingua e della poesia di Petrarca e dei suoi epigoni; e come spesso accade, il lessico nobile e raffinato che si insinua nei ritmi dell'endecasillabo e nella memoria letteraria di poeti dilettanti e professionisti, nonché di tutti coloro che nel corso del Cinquecento accedono alla lirica, diventa anche uno degli strumenti di sostegno per l'approfondimento e la pratica di una lingua straniera. Se, senza dubbio, nel caso dell'apprendimento dello spagnolo da parte degli italiani, vista l'occupazione e le esigenze politiche e amministrative ad essa

\footnotetext{
${ }^{1}$ Tempio alla divina signora donna Giovanna d'Aragona, fabricato da tutti i più gentili spirti, \& in tutte le lingue principali del mondo, Venezia, Plinio Pietrasanta, 1555.

${ }^{2}$ Rime et versi in lode della ill.ma et ecc.ma S.ra D.na Giovanna Castriota Carrafa, duchessa di Nocera et marchesa di Civita S. Angelo. Sscritti in lingua Toscana, Latina, et Spagnuola da diversi huomini illust. in varij, et diversi tempi, et raccolti da Scipione de Monti, Vico Equense, G. Cacchi, 1585.
} 
dovute, va messa in luce in tutta la sua importanza la dimensione orale e il criterio dell'uso come elemento di quotidiano esercizio e dinamica verifica sul campo, tuttavia siamo convinti che per questi poeti sia altrettanto significativa l'acquisizione e la pratica della lingua scritta, e in particolare dello spagnolo letterario: non crediamo infatti che sia casuale che nelle principali città degli stati assimilati, proprio nei decenni centrali del XVI secolo, vengano pubblicate varie edizioni di libri in castigliano - libri completi di paratesti consistenti in dizionari e glossari o integrati da altri strumenti lessicografici di pronto utilizzo per il lettore italiano ${ }^{3}$ - nonché le prime vere e proprie grammatiche spagnole ad uso degli italiani, all'interno delle quali gli esempi principali erano tratti tanto dalla lingua parlata quanto, e anzi soprattutto, da quella scritta, dalla letteratura ${ }^{4}$. Il sistema della comunicazione petrarchistica si presenta come un sistema sovralinguistico, dotato prima di tutto di un repertorio canonizzato di forme metriche, elementi stilistici e lessicali rigorosamente selezionati da riusare indipendentemente dall'idioma dei parlanti (e degli scriventi); e tanto la lingua spagnola quanto quella italiana - ma un discorso simile si potrebbe fare probabilmente anche per quella francese, portoghese, inglese, tedesca ecc. in questo senso dimostrano di "funzionare" agevolmente all'interno delle sue strutture. Le architetture - formali e spirituali: imitatio styli vitaeque - del petrarchismo in questo senso sembrano garantire una sorta di ipotesto di sicura affidabilità ed eleganza, che i poeti di ogni tradizione letteraria e linguistica europea collaborano a modulare e sviluppare a

\footnotetext{
${ }^{3}$ Ricordiamo qui principalmente i due dizionari/glossari curati proprio all'inizio degli anni cinquanta da Alfonso de Ulloa: la Exposición de algunos vocablos Castellanos en lengua Thoscana, inserita al termine dell'Orlando furioso de m. Ludovico Ariosto... traduzido en romance castellano por el S. Don Hieronimo de Vrrea Venecia, por Gabriel Giolito de Ferrariis y sus hermanos, 1553, pp. 533-615 (non numerate); nonché laIntrodutione del Signor Alfonso di Uglioa nella quale s'insegna pronunciare la lingua Spagnuola, con una espositione da lui fatta nella Italiana, di parecchi vocaboli Hispagnuoli difficili che chiude la sua edizione della Celestina: Tragicomedia de Calisto y Melibea, Venezia, por Gabriel Giolito de Ferrariis y sus hermanos, 1553. In ogni caso i due dizionari di Ulloa, che vennero riproposti anche in altre edizioni successive, potevano contare su alcuni occasionali precedenti, tra cui forse il più significativo rimane quello di Francisco Delicado, che si era soffermato sulla spiegazione della pronuncia del castigliano nelle Advertencias poste al termine della sua edizione della Celestina (Venezia, Giolito, 1534). Comunque, per altre notizie e approfondimenti su questi paratesti e per ulteriori rimandi, cfr. direttamente J. M. Lope Blanch, Estudios de historia lingüística hispánica, Madrid, Arco/Libros, 1990, p. 27.

${ }^{4}$ La prima grammatica castigliana ad uso degli italiani fu quella redatta da Giovanni Mario Alessandri, che tra l'altro aveva vissuto alcuni anni alla corte di Spagna: Il Paragone della lingua toscana et castigliana, Napoli, Mattia Cancer, 1560. Tuttavia il maggiore interprete dell'insegnamento dello spagnolo in Italia è senz'altro Giovanni Miranda, le cui Osservationi della lingua Castigliana (Venezia, Giolito, 1566) ebbero grande successo e furono ripubblicate varie volte nel secolo. Sempre Lope Blanch, infatti, definisce 1'opera di Miranda «la más completa $\mathrm{y}$, al mismo tiempo, la más equilibrada, práctica y provechosa de cuantas se publicaron por aquel entonces», e ancora «el mejor manual para la enseñanza sistemática y científica del español a los italianos», al punto che il suo testo venne imitato da molti grammatici stranieri, francesi e inglesi soprattutto. Per questo discorso, cfr. ancora, J. M. Lope Blanch, Estudios de historia lingüística hispánica, cit., p. 28 n. 71, ma anche A. Ramajo Caño, Las gramáticas de la lengua castellana desde Nebrija a Correas, Salamanca, Universidad de Salamanca, 1987, in particolare p. 236. Su Miranda esiste comunque una discreta bibliografia, tra cui vale qui la pena ricordare almeno gli studi specifici di M. Carreras i Goicoechea, "Giovanni Miranda i les primeres temptatives d' ensenyament del castellà a Itàlia", in Anuari de Filologia, XV, 1992, sèrie G, 3, 77-86; 'Esiste una “coscienza lessicografica in Giovanni Miranda?”, in Boletín de la Real Academia de Buenas Letras, XLIV, 199394, pp. 427-446; Le fonti delle Osservationi della lingua castigliana di Giovanni Miranda, in M. Tavoni, a c. di, Italia ed Europa nella linguistica del Rinascimento, Modena, Panini, 1996, pp. 475-493; e infine il recente "El papel de las Osservazioni della lingua castigliana de Giovanni Miranda (1566) en la historia de la enseñanza del español para italianos", in Id., Cagnolati - Vicentini et al., Storia degli insegnamenti linguistici: bilanci e prospettive. Atti del Convegno di Bologna, 15 novembre 2002, Quaderni del CIRSIL, Bologna, Clueb, 2005 , pp. 9-23.
} 
proprio piacimento, ampliandone così allo stesso tempo gli stessi orizzonti culturali. Non è questa la sede per affrontare in maniera precisa, con esempi dettagliati, la "transitività" linguistica del petrarchismo, cioè la capacità di mutuare, sostituire o riadattare un termine, una formula da un idioma a un altro, che pure si avverte nella tradizione lirica italiana e spagnola all'interno di uno o più metri consacrati; tuttavia proprio l'abilità dei rimatori e, paradossalmente, la ripetitività di esperimenti poetici in "lingua seconda" nonché, su un piano più generale, la crescente fortuna editoriale di progetti antologici plurilingue - ma a parte le lingue antiche (latino, greco), in Italia l'alternanza rimane per lo più tra italiano e castigliano - confermano a tutti gli effetti che nel secondo Cinquecento la poesia italiana e spagnola, o meglio la lingua della loro poesia, tanto sul fronte del lessico quanto su quello del ritmo, obbedisce di buon grado al medesimo codice. Il mondo editoriale, quindi, dal canto suo, non fa altro che rispondere a sua volta al corso degli eventi storici, alle opportunità commerciali e ai gusti del pubblico: in genere, in tutto il continente europeo, da Venezia ad Anversa a Valencia, si moltiplicano canzonieri e antologie di diversi autori e in diverse lingue, spesso compresenti, che vivono tutti delle possibilità linguistiche, sociali ed economiche offerte dalla rete del petrarchismo internazionale. In un'Europa in cui, a metà Cinquecento, il sogno di un'unità sia politica che religiosa è destinato a tramontare di fronte alle inconciliabili posizioni emerse nel corso del Concilio di Trento, proprio il petrarchismo - inteso come pratica linguistica, letteraria e sociale ma anche, e soprattutto, comebusiness di prim'ordine per l'industria tipografica - sembra rimanere l'unico codice di relazione a cui la società europea accetti di aderire pienamente. Dalla Gran Bretagna alla Spagna, dai Principati tedeschi allo Stato pontificio, insomma in tutta l'Europa di Antico Regime, e - lo ripetiamo - specialmente in seguito alla dolorosa e insanabile cesura costituita dalla redazione della confessio fidei tridentina, l'unica icona credibile e condivisibile a livello internazionale non è più quella del Papa o dell'Imperatore, bensì quella di Francesco Petrarca. E di conseguenza, di fronte alla barbarie delle devastazioni belliche e al fondamentalismo di Cattolici e Riformati, l'unico "catechismo" riconosciuto dagli intellettuali europei rimarrà quello dei Rerum Vulgarium Fragmenta.

L'utilizzo della lingua spagnola da parte dei poeti italiani del secondo Cinquecento, in ultima analisi, non può essere ridotto né a un dotto e accademico esercizio né a un semplice omaggio alla tradizione linguistica d'origine delle personalità lodate, siano esse Giovanna d'Aragona, Giovanna Castriota o qualche altra dama legata all'aristocrazia ispanica in Italia: comporre liriche direttamente in castigliano non soltanto dimostra la volontà da parte di questi autori italiani di ostentare la propria competenza linguistica, cimentandosi in una lingua non materna, e la devozione verso i dominatori (anzi, nel frangente, verso le mogli dei propri comandanti, principi o datori di lavoro), ma più che altro testimonia, di fatto, come questi letterati, quotidianamente a contatto per ragioni professionali con l'intelighenzia spagnola sul territorio, adoprino la «castellana lengua» come uno strumento linguistico d'uso concreto e non come un elemento estraneo alla propria cultura. Inoltre, dopo decenni di Renacimiento, la lingua spagnola aveva dimostrato anche in Italia il suo peso e la sua dignità sul piano strettamente letterario - basti pensare al caso del "napoletano" Garcilaso in poesia, ma anche a tutta l'importanza e al successo editoriale della narrativa cavalleresca e "celestinesca" - forzando così i limiti che inizialmente

\footnotetext{
${ }^{5}$ Una breve ricognizione nella tipografia italiana del Cinquecento permette di contare varie edizioni tanto dei libri più siginificativi della tradizione della «caballería» quanto della poesia di Garcilaso o dellaCelestina. Ricordiamo qui, in primo luogo, le due edizioni italiane delle Obras di Boscán e Garcilaso: Las obras de Boscan y algvnas de
} 
l'avevano fatta considerare dai letterati più conservatori, per ovvie ragioni storiche e politiche, più che altro come la lingua della milizia e della diplomazia. Non c'è dubbio infatti che $\mathrm{i}$ sonettisti italiani che desiderano provare la propria «pluma» nell'idioma di Castiglia, negli anni della compilazione del Tempio e soprattutto in quelli delle successive Rime et versi, abbiano ormai a disposizione più di un modello da imitare nell'ambito della tradizione letteraria spagnola: tra i rimatori «a la manera italiana», su tutti, lo stesso Garcilaso; ma non va neppure dimenticato tutto il movimento di poeti-soldati iberici della stessa generazione, da Gutierre de Cetina a Hernando de Acuña, riuniti dapprima presso Alfonso d'Avalos nei terrirori del Milanesado e poi dispersisi al seguito dei propri reggimenti tra i vari stati italiani, l'Africa mediterranea e perfino il Nuovo Mondớ. Le liriche in castigliano che un letterato attento e preparato come Girolamo Ruscelli introduce nel Tempio esemplificano senza dubbio un uso calcolato del lessico di ascendenza petrarchesca; e anzi, proprio il fatto che i responsabili dei versi siano spesso autori italiani "travestiti" - nel senso che, come osserveremo, il più delle volte essi "ispanizzano" non solo la propria poesia ma anche i propri nomi - fa sì che queste composizioni appaiano meno soggette a "ricadute" cancioneriles. Con questi poeti in "lingua seconda" siamo perciò lontani ormai, nei decenni del secondo Cinquecento, dal clima di curiosità erudita ed esotica che contraddistingueva ancora, ad esempio, gli occasionali tentativi di Bembo di comporre alcune coplas alla castigliana per compiacere Lucrezia Borgia?: i poeti selezionati

Garcilasso de la Vega Repartidas en quatro libros. Ademas que ay mvchas anadidas uan aqui mejor corregidas, mas coplidas y en mejor orden que asta agora han sido impressas, Roma, M. Antonio de Salamanca, 1547; e Las obras de Boscan, y algvnas de Garcilasso de la Vega A demas qve ay mvchas añadidas, uan aqui mejor corregidas, mas complidas y en mejor orden que hasta agora han sido impressas Venezia, Giolito, 1553. Tra $\mathrm{i}$ libri di cavalleria, ad esempio, l'Amadís de Gaula fu pubblicato invece ... [continua]. Anche della Celestina esistono sia edizioni in castigliano che in italiano: ...

${ }^{6}$ Emblematico il caso del sivigliano Gutierre de Cetina, che dopo aver trascorso gli anni più importanti della sua carriera militare e della sua formazione umana e poetica tra l'Italia e l'Africa del Nord si trasferì a Città del Messico, dove morì intorno al 1557. Durante gli anni messicani, Cetina fu uno dei mediatori più importanti del petrarchismo italiano nel Nuovo Mondo. Su quest'ultimo aspetto, cfr., tra gli altri, soprattutto G. Bellini, Presencia de la literatura italiana en la America Hispana de los siglos XVI y XVIL in AA. VV., España e Italia: un encuentro de culturas en el Nuevo Mundo. Atti del colloquio italo-spagnolo (Barcellona, 20-22 aprile, 1989), Roma, Bulzoni, 1991, pp. 37-57; e, dello stesso autore, il precedente Storia delle relazioni letterarie tra l'Italia e l'America di lngua spagnola, Milano, Cisalpino-Goliardica, 1977; ma anche A. Peconi, "Libri e stampatori italiani nella Nuova Spagna nel secolo XVI", Quaderni Ibero-americani, 51-52, 1978-79; e E. Núñez, Las letras de Italia en el Perú, Lima, Universidad Nacional de S. Marcos, 1968.

${ }^{7}$ I versi spagnoli di Pietro Bembo, risalenti al 1503, e perciò agli anni degliAsolani e del più intenso legame con Lucrezia, al di là del contesto amoroso, appaiono una semplice dimostrazione di abilità versificatoria e virtuosismo linguistico e non rappresentano comunque un reale interesse del poeta veneziano per la poesia della tradizione castigliana; sono invece più che altro testimonianza di una corte ferrarese - quella di Ercole I, la cui moglie era Eleonora d'Aragona - in cui l'influenza delle nobildonne ispaniche si faceva sentire sia a livello mondano che letterario. L'autografo che contiene questi versi si trova nella Biblioteca Ambrosiana (Ms.S.P.II.100) e trascrive i versi bembiani nonché quelli «in risposta» redatti dalla stessa Lucrezia Borgia: in pratica, dunque, quella tra i due fu una corrispondenza amorosa in perfetto stilecancioneril. Alcune strofe di Bembo furono pubblicate in varie raccolte delle sue opere, mentre i versi della Borgia vennero invece pubblicati a parte all'interno dell'edizione delle lettere indirizzate al Cardinale: B. Gatti,Lettere di Lucrezia Borgia a messer Pietro Bembo dagli autografi, Milano, Tip. dell'Ambrosiana, 1859. Su questo carteggio poetico rimangono comunque fondamentale gli studi di Teza, Versi spagnoli di Pietro Bembo, a cura di E. Teza, Livorno, Tip. di Francesco Vigo, 1882; e di P. Rajna, I versi spagnoli di mano di Pietro Bembo e di Lucrezia Borgia serbati da un codice ambrosiano, in AA.VV., Homenaje ofrecido a Ramón Menéndez Pidal, Madrid, Hernando, 1925, vol. II, pp. 229-321. Comunque, per i rilievi filologici e per tutti i riferimenti bibliografici, cfr. soprattutto B. Croce, Cose spagnuole alla corte di Ferrante, in Id., La Spagna nella vita italiana durante la Rinascenza, Bari, Laterza, 1917, pp. 65-67; e G. Mazzocchi, Un manoscritto milanese (Biblioteca Ambrosiana S.P.II.100) e l'ispanismo del 
da Ruscelli, così come Scipione de Monti e i suoi colleghi nelle Rime et versi per Giovanna Castriota, pur con tutte le riserve del caso, utilizzano lo spagnolo in quanto lingua letteraria a tutti gli effetti, una lingua che vantava da alcuni decenni opere significative alla luce dell'intera stagione rinascimentale europea e che, sul fronte specifico della poesia lirica dimostrava di essersi saputa integrare perfettamente nelle strutture ritmiche e spirituali del petrarchismo.

Uno dei primi e più significativi casi della compresenza, all'interno di un'antologia del secondo Cinquecento, di autori che scrivono tanto in italiano quanto in castigliano è costituito dunque dal Tempio per Giovanna d'Aragona, uscito per i tipi di Plinio Pietrasanta nel 1555. In quanto alle presenze all'interno della miscellanea, la proporzione non è omogenea e i poeti italiani, o quelli che comunque scrivono in lingua italiana, sono molto più numerosi dei loro omologhi spagnoli, tuttavia le ultime pagine accolgono proprio una microsezione che racchiude alcune liriche in castigliano composte per lo più da autori italiani. Come vedremo, dietro i nomi ispanizzati della maggioranza di questi poeti si nascondono (ma non troppo) alcune personalità dell'aristocrazia italiana vicina alle «cose» di Spagna e legata alla famiglia degli Aragona, nonché alla monarchia asburgica, da non poche ragioni di natura culturale, politica ed economica: sul piano linguistico, l'uso della lingua castigliana, che rappresentava ovviamente il codice principe delle relazioni con i dominatori iberici, costituiva in se stessa un ossequio e un riguardo nei confronti di questi ultimi, che avrebbero così gustato le lodi riservate alla «divina» Giovanna nell'idioma natio; sul fronte dell'opportunità politica, invece, l'ammirazione e l'omaggio per la nobile casata iberica, o meglio per i discendenti di essa, che ricoprivano posizioni di prestigio sociale e incarichi amministrativi di rilievo nell'Italia occupata, probabilmente servivano a garantire a questi più o meno improvvisati rimatori il mantenimento e il consolidamento dei propri privilegi e benefici nei vari territori della penisola.

La raccolta, a cura di Girolamo Ruscelli, rappresenta -come ha mostrato Quondam«uno spaccato sincronico di straordinaria efficacia documentaria sulle proporzioni stesse dell'accesso al petrarchismo nella società cinquecentesca, sulle sue modalità d'esercizio, sulle sue funzioni comunicative» ${ }^{8}$. L'intenzione di fondo dell'operazione è dunque, fin dal titolo, scopertamente encomiastica: Giovanna d'Aragona è infatti un personaggio di spicco dell'aristocrazia italospagnola in Italia, e i vari autori inlcusi nella raccolta rispondono

Bembo, in G. Caravaggi, (a cura di), Cancioneros spagnoli a Milano, Firenze, La Nuova Italia, 1989, pp. 67-100; mentre, per i dettagli cronologici e biografici della relazione, basti menzionare un saggio datato ma con numerosi spunti: B. Morsolin, "Pietro Bembo e Lucrezia Borgia", Nuova Antologia, LII, 1885, pp. 388-422.

${ }^{8}$ A. Quondam, Il naso di Laura: lingua e poesia lirica nella tradizione del classicismo, Modena, Panini, 1991, p. 135. Per le notizie dettagliate sulla nobildonna italospagnola, cfr., direttamente, ivi, p. 148, n. 46; mentre, in generale, per ulteriori ragguagli sulla raccolta in questione, cfr. sempre ivi, pp. 132-150. AltriTempi, comunque, appaiono nella seconda metà del Cinquecento: basti ricordare il noto Tempio della divina signora donna Geronima Colonna d'Aragona, Padova, Pasquati, 1568, dedicato appunto alla figlia della stessa Giovanna d'Aragona e di Ascanio Colonna; ma anche, significativo dell'attenzione riservata a personaggi di località specifiche, il Tempio fabricato da diversi coltissimi, \& nobiliss. Ingegni, in lode dell'illust.ma \& ecc.ma donna Flavia Peretta Orsina, ducchessa di Bracciano, Roma, G. Martinelli, 1591. Per un approfondimento sulla tipologia della raccolta encomiastica, cfr., tra gli altri, anche il recente studio di F. Tomasi,Alcuni aspetti delle antologie liriche del secondo Cinquecento, in "I più vaghi e i più soavi fiori». Studi sulle antologie di lirica del Cinquecento, a cura di Monica Bianco ed Elena Strada, Alessandria, Edizioni dell'Orso, 2001, in particolare pp. 100-108, e quello più specifico di M. Bianco, Il "Tempio" a Geronima Colonna d'Aragona ovvero la conferma di un archetipo, ivi, pp. 147-181. 
organicamente alle esigenze progettuali. È perciò evidente un'unità di intenti riconoscibile alla base dell'antologia:

Tutti i testi del Tempio svolgono lo stesso, unico, predeterminato tema; l'insieme è frantumato, polverizzato in una miriade di testi unici, che non consentono di definire in alcun modo la presenza dell' "autore", ma che trovano invece specifica determinazione semantica nel solo fatto di trovarsi dentro il "tempio", come segmenti necessari seppur intercambiabili, dell'edificio complessivo, che attribuisce però a ciascuno di essi la possibilità di significare, orientandone a senso unico la disponibilità tematica e persino lessicale. ${ }^{9}$

Nella diffusione del petrarchismo in Spagna, o comunque nell'universo letterario spagnolo, i progetti miscellanei di carattere celebrativo rappresentarono senza dubbio un canale particolarmente agevole: la lode poetica di un personaggio del calibro di Giovanna d'Aragona non solo meritava di essere conosciuta e di circolare nei territori della penisola iberica, ma era da considerarsi a tutti gli effetti un esempio da seguire e imitare; e inoltre questo tipo di poesia, occasionale e stereotipata, offriva un primo, rapido strumento per l'iniziazione di letterati e uomini di palazzo spagnoli alle «regole» e ai dettami - metro, stile, lessico, topica - della lirica petrarchista. In generale, a proposito delle svariate e molteplici iniziative encomiastiche, che sul modello del Tempio videro la luce in Italia nel secondo Cinquecento, si può mettere in evidenza una duplice caratteristica piuttosto ricorrente: dal lato degli occasionali dedicatari, che poi per lo più sono dedicatarie ${ }^{10}$, la presenza notevole di mandatari e dame di provenienza o quantomeno parentela iberica ribadisce la posizione di predominio che i nobili di origine castigliana ricoprirono nelle questioni diplomatiche, militari, o anche solo nello spaccato sociale degli stati italiani, e conferma che proprio per questa ragione essi meritavano un elogio appropriato, quale solo la poesia lirica poteva assicurare e - grazie alle risorse della stampa - amplificare; dall'altro lato, quello dei "dedicatori", i vari curatori furono i primi a favorire in quegli stessi progetti, per ragioni commerciali e "politiche", la presenza sia pur minima di rime e rimatori spagnoli che, pur essendo inferiori per quantità e qualità rispetto ai corrispettivi italiani, venivano di fatto accolti e omologati, insomma consacrati, all'interno del nobile universo del petrarchismo. Appare pertanto perfettamente comprensibile la disponibilità da parte degli addetti ai lavori dell'industria tipografica a inserire nel mercato letterario, italiano e spagnolo, a diverso titolo (dedicatario, prefatore o rimatore), membri più o meno influenti dell'intellettualità iberica in Italia, e ciò avviene tanto per le menzionate ragioni di opportunità commerciale quanto anche per l'estensione e la diffusione della consuetudine letteraria $^{11}$

\footnotetext{
${ }^{9}$ Ivi, pp. 136-137..

${ }^{10}$ Quondam: «La preminenza di un soggetto femminile consegue sia dalla funzionalità strettamente cortigiana di questa celebrazione, sia dall'impianto costitutivo del genere lirico, destinato alla lode di una donna (amata)», ivi, p. 149, n. 52. Come abbiamo già accennato, il fenomeno delle dediche ad ambasciatori e governatori di Sua Maestà è tutt'altro che eccezionale e un'indagine su quest'ultimo aspetto meriterebbe una ricerca a sé. Ad ogni modo, per ciò che concerne le fínalità che più o meno scopertamente risiedono dietro l' "offerta" di questa o quell'opera, la già ricordata dedica della prima edizione delle Rime diverse, a cura del Domenichi, all'ambasciatore nonché poeta petrarchista don Diego Hurtado de Mendoza parla chiaro: questa raccolta si pubblica a Venezia negli anni in cui l'illustre dignitario vi ricopre, per conto del Cesare, proprio la più alta carica diplomatica. In questa maniera, il venerando dedicatario se, da una parte, riceve ulteriore lustro e gloria per sé grazie al libro, dall'altra, diventa anche un potente alleato per la garanzia e il successo dell'editore.
} 
Nella sezione spagnola del Tempio così come in quella presente nelle Rime et versi, ma in generale in più di una miscellanea in lode di questa o quella dama, non si trovano comunque, come accennavamo in precedenza, i grandi nomi della letteratura castigliana e della corrente italianizante, ma muove più che altro i primi passi una schiera di petrarchisti, più o meno occasionali e "sotterranei", accomunati tutti però dall'uso della lingua spagnola: all'altezza della seconda metà del Cinquecento ufficiali, dignitari, letterati e nobildonne ${ }^{2}$ iberici partecipano dunque attivamente alle funzioni, per la verità più sociali che culturali, della lirica del tempo e si inquadrano senza alcun impaccio nell'universo ormai linguisticamente frastagliato, eppure unitario, della comunicazione petrarchistica. E accanto a questi "esordienti", sul fronte delle relazioni tra la lingua spagnola e quella italiana nel corso del XVI secolo, il fenomeno più interessante riguarda senza dubbio la presenza di diversi poeti italiani, i quali, "camuffati" dietro improbabili appellativi spagnoleggianti, che poi per lo più si riducono all'ispanizzazione del nome, per le ragioni elencate in precedenza redigono in castigliano i propri sonetti di dedica e di encomio. A conferma del contatto strettissimo, della contiguità quasi, tra la lingua e la cultura italiana e spagnola, che si verifica in questi anni tanto sul versante storico e geopolitico quanto, soprattutto, nei territori del petrarchismo, proprio Ruscelli, nell'epistola introduttiva al Tempio, osserva come la schiera di «tanti begli ingegni» impegnati a cantare Giovanna d'Aragona rappresenti «una provincia tutta (che tutto un mondo si potrà ben dir tosto)»» ${ }^{13}$. E nella successiva epistola Ai Lettori, che segue la dedicatoria vera e propria e che desidera spiegare i (quattro) criteri con cui è stata composta la raccolta, il curatore sottolinea ciò che veniva già annunciato nel titolo, e cioè il fatto che i testi provengono da «tutti i più famosi ingegni dell'età nostra» e che appaiono «in tante lingue»" ${ }^{14}$, senza desiderare stabilire al contempo alcuna gerarchia o graduatoria: per il nostro discorso, lasciando da parte il latino e il greco, tanto l'italiano quanto il castigliano sembrano meritare la medesima considerazione purché partecipino attivamente, in particolare, all'elogio incondizionato della «divina» Giovanna e, in generale, al circuito internazionale del petrarchismo. E infine, sempre in queste pagine, come a ribadire l'ingombrante presenza - politica, sociale,

\footnotetext{
11 Possono ampliare e completare quanto detto finora alcuni rilievi del curatore Girolamo Ruscelli inseriti nell'epistola introduttiva. Nelle prime pagine della dedicatoria, dopo un generico discorso sulla superiorità della «favella» o della «scrittura», con il primato di quest'ultima perfettamente inquadrato nel repertorio delle dispute cinquecentesche, si richiama come doverosa la lode riservata alla nobiltà dei Principi, tra cui ovviamente la destinataria del Tempio: Ruscelli è infatti il primo a riconoscere «i gran meriti, \& il sommo valore, \& la bellezza infinita di corpo, \& d'animo della Illustrissima \& Eccellentissima Signora DONNA GIOVANNA D’ARAGONA». Cfr. G. Ruscelli, All'Illustrissimo, et Reverendiss. Signore, il Signor Cristoforo Madruccio, Cardinal di Trento, in Il Tempio alla divina signora donna Giovanna d'Aragona, Fabricato da tutti i più gentili spirti, \& in tutte le lingue principali del mondo, Venezia, «per Francesco Rocca, à S. Polo all'insegna del Castello», 1565, c. 7v. Citiamo sempre da questa edizione.

12 Come avviene anche nel caso italiano, il petrarchismo costituisce il primo momento in cui è ampiamente documentabile nell'universo spagnolo il fenomeno di una lirica "al femminile". Con l'introduzione delle teorie cortigiane in Spagna, la donna assume la stessa dignità dell'uomo e partecipa con lui al gioco sociale che si tiene a Palazzo; e il XVI secolo annovera altresì le prime donne che frequentano le università e approfondiscono gli studia humanitatis. Dunque, proprio per queste ragioni la stragrande maggioranza delle liriche al femminile nella Spagna del XVI secolo ha come argomento privilegiato la celebrazione delle vicende di corte (matrimoni, morti, nascite ecc.). Cfr. A.Navarro, a c. di, Introducción a Antología poética de escritoras de los siglos XVI y XVII, Madrid, Castalia, 1989.

${ }^{13}$ G. Ruscelli, All'Illustrissimo, et Reverendiss. Signore, il Signor Cristoforo Madruccio, Cardinal di Trento in Il Tempio alla divina signora donna Giovanna d'Aragona..., cit., c. 8r.

${ }^{14}$ G. Ruscelli, Ai lettori, in Il Tempio alla divina signora donna Giovanna..., cit., c. 19r.
} 
linguistica - della nazione spagnola in Italia, lo stesso Ruscelli conclude la sua epistola riconoscendo apertamente la grandezza della Spagna imperiale: «Senza che si dee tener per fermo, che la nobilissima, \& in ogni sua cosa pienamente honoratissima natione SPAGNUOLA, dalla quale per casa di Re questa gran Signora trae origine, sia per far miracoli, \& per non cedere à niuna dell'altre, né anco all'ITALIA stessa ${ }^{15}$.

Le rime in castigliano nel Tempio, nonostante il loro esiguo numero, offrono un campionario abbastanza vario di metri e versi tanto «a la manera italiana» quanto cancioneril $^{16}$. Riportiamo di seguito la lista dei vari componimenti:

\begin{tabular}{|l|l|l|}
\hline Rinaldo Corso & Quien es esta Diosa? & Madrigale \\
Iusepe de Cerros & Ruscel fue el arquitecto & Canzone \\
Odoardo Gomez & El Rey del todo en la fazon primera & Sonetto \\
& Illustre dama, en quien puso natura; & Sonetto \\
Iuan Garçia de Brinag & Gran impetu me toma, y gran desseo & Sonetto \\
Ginez de Canizares & Lo mas que yo puedo señora dezir & Ottave di arte mayor \\
HernandoAfrodiseo d'Aragon & Alla' en Montalto, de donde deriua & Ottava rima \\
Francisco Alonso & De donde podré tomar yo'l dechado & Ottave di arte mayor \\
& Quien me darà la boz, dama excelente; & Sonetto \\
Iuan Batista Brembado & Si alguna tal salio, qu'es marauilla & Sonetto \\
Marsilio de la Cruz & Sabio Ruscel, que à tan alta Diosa & Sonetto \\
\hline
\end{tabular}

Come si può osservare dall'elenco, alcuni autori tradiscono immediatamente la propria origine italiana e perciò sembrano confermare in modo emblematico come nella loro coscienza linguistica il castigliano non dovette limitarsi soltanto, o prevalentemente, al ruolo di lingua d'uso "quotidiano", linguaggio privilegiato della milizia, del banchetto e della burocrazia; al contrario, appare evidente, almeno in questa sede, cioè all'interno della raccolta ruscelliana -ma stesso discorso si potrebbe fare per le Rime et versi di Scipione de Monti-, come questi rimatori italiani utilizzino lo spagnolo a tutti gli effetti come una lingua letteraria. E il codice di riferimento, che realmente permette loro di districarsi abilmente nella «universal castellana lengua», è quello proprio del petrarchismo: le scelte in fatto di lessico, il computo sillabico e l'andamento ritmico dei vocaboli spagnoli rispondono appieno alle esigenze metriche e stilistiche della «manera italiana» così come essa si andava esercitando in Spagna da vari decenni. Questi poeti, dunque, nel momento di comporre sonetti e canzoni in castigliano, da una parte, ebbero presenti, ovviamente, i modelli italiani internazionalmente celebrati e acquisiti nell'ambito dei vari "petrarchismi" nazionali Petrarca, ma anche Bembo, Ariosto, Sannazaro ecc. -; dall'altra, però, dovettero apprendere molto, sul piano lessicale e retorico, anche e soprattutto dalle prime manifestazioni della lirica petrarchista spagnola - Boscán e Garcilaso su tutti - che, in virtù del contatto costante

\footnotetext{
${ }^{15}$ Ivi, c. 22 r.

${ }^{16}$ Cfr. sempre l'edizione veneziana del 1565. Le differenze rispetto all'edizione menzionata e analizzata da Quondam riguardano principalmente il numero dei rimatori di lingua spagnola: lì risultano sette per un totale di otto liriche; qui invece i poeti che scrivono in castigliano sono dieci, per un totale di dodici composizioni (di cui due in metro castigliano, quelle di García de Brinag e di Afrodiseo d'Aragón, e dieci «a la manera italiana»).
} 
tra la penisola italiana e quella iberica e grazie soprattutto alle strategie commerciali dell'industria tipografica, circolarono presto e in modo diffuso nei territori imperiali in Italia.

Rinaldo Corso (1525-1580 o 1582) $)^{17}$, ad esempio, compare con il proprio "italianissimo" nome tanto nella sezione dei rimatori italiani, dove è presente con il madrigale Come nessuna donna, quanto in quella spagnola, e conferma la sua attitudine poliglotta e la sua abilità poetica anche nella parte latina, dove figura, come Rainaldus Corsius, con il distico Hoc tibi Castalides ponunt Aragonia Templum. Senza dubbio, comunque, l'abilità letteraria di Corso non si limitò a queste occasionali prove: veronese di nascita, fu un giurista e un letterato piuttosto attivo nei territori dell'Italia nordorientale durante i decenni centrali del secolo e partecipò alle iniziative del circolo di Veronica Gambara a Correggio, offrendo in più di un'occasione testimonianze convincenti della propria competenza filologica, linguistica e grammaticale. A Venezia pubblicò infatti i Fondamenti del parlar thoscano (Venezia, Comin da Trino, 1549), che come grammatica riscossero un certo successo, viste le cinque ristampe che l'opera conobbe nel corso del secolo; tradusse le Pastorali di Virgilio (Ancona, 1564) e si occupò a livello filologico e critico della lirica di Vittoria Colonna, circostanza che non sfuggì all'attento Ruscelli1 ${ }^{18}$; come poeta, invece, dette alle stampe una raccolta di Rime di scarsa circolazione (Bologna, Muzio Manfredi [?], 1575). Infine, sul fronte biografico, dopo aver avuto simpatie luterane o comunque eterodosse, abbracciò lo stato ecclesiastico, avvicinandosi in modo netto alle posizioni politico-religiose della Spagna post-tridentina e giungendo perfino ad essere inquisitore: da qui alcuni suoi componimenti in terza rima di argomento dottrinario inseriti dal Possevino nel suo Soldato cristiano (Mantova, 1583). In ordine di apparizione viene poi Iusepe de Cerros, al secolo Giuseppe Cerri, il quale si dimostra come gli altri un brillante versificatore e un sapiente poliglotta, dato che prima della sua lirica in spagnolo appaiono nelle ultime pagine del volume di Ruscelli anche un componimento in tedesco e uno in italiano. Le sue simpatie filospagnole si riflettono, inoltre, al di là dei componimenti inseriti in questa antologia, nella sua Canzone nel passaggio delli sereniss. principi d'Austria Rodolfo et Hernesto di Germania nella Spagna, l'anno MDLXIII (Bressanone, Donato Feccio, 1564). La peculiare tendenza a redigere la lode poetica in più lingue si manifesta, tra gli altri, anche nel caso del conte Giovan Battista Brembati (1509-1573) -qui ispanizzato come «el Conde Iuan Bautista Brembado»-, che per altro offre la testimonianza più significativa ed emblematica, da un lato, dei legami che i rimatori del Tempio dovettero avere con la nobile casata d'Aragona e in generale con la monarchia iberica e i suoi rappresentanti in Italia, dall'altro, del criterio di scelta dei diversi autori utilizzato da Ruscelli per la formazione della raccolta. Brembati, bergamasco di nascita, si dedicò infatti fin dalla giovinezza al mestiere delle armi e servì la Corona spagnola agli ordini del Duca

\footnotetext{
${ }^{17}$ In generale, per le notizie biografiche e i riferimenti editoriali relativi agli autori del Tempio, rimandiamo diffusamente al Dizionario Biografico degli Italiani, ...; e al repertorio di informazioni biobibliografiche fornito da EDIT 16 - Censimento nazionale delle edizioni cinquecentesche presenti nelle biblioteche italiane (risorsa elettronica a cura del Servizio Bibliotecario Nazionale: www.sbn.it).

${ }^{18}$ Girolamo Ruscelli, pochi anni dopo la pubblicazione del Tempio, curò proprio l'edizione di Tutte le rime dell'illustriss. et eccellentiss. signora Vittoria Colonna Marchesana di Pescara, con la esposizione del signor Rinaldo Corso (Venezia, Sessa, 1558). In realtà, a testimonianza della sua antica passione per la lirica della Colonna, Corso aveva già composto in precedenza una Dichiaratione fatta sopra la Seconda Parte delle Rime della divina Vittoria Colonna, Bologna, G. B. Faelli, 1543, dedicata a Veronica Gambara e comprensiva di due sonetti dello stesso autore.
} 
d'Alba e del Duca di Sessa, raggiungendo negli eserciti imperiali il grado di colonnello; in questo modo godette sempre della protezione spagnola e fu grazie alle sue influenti amicizie che riuscì a condurre in porto un'esistenza caratterizzata per lo più da feroci faide nobiliari tra Milano, Bergamo e Venezia (in suo favore si pronunciarono in un paio di casi perfino Giovanni d'Austria e lo stesso Filippo II). Sul fronte letterario, invece, dovette distinguersi positivamente nella poesia lirica, dal momento che un letterato raffinato come Ruscelli non solo lo inserì nella miscellanea per Giovanna d'Aragona ma, pochi anni dopo, incluse alcune sue liriche nella fortunata antologia dei Fiori delle rime de' poeti illustri (Venezia, Sessa, 1558) e, a conferma della stima che comunque nutrì nei suoi confronti, gli dedicò altresì un'edizione da lui curata del Decameron (Venezia, 1552). Tra gli altri, ricordiamo infine un importante e affascinante protagonista delle vicissitudini della dominazione spagnola in Italia: Cesare Carafa. Campano e figlio del conte Diomede, lui e la famiglia subirono le ire, le confische e le condanne di don Pedro de Toledo in quanto vicini a Ferrante Sanseverino, capo dei moti contro l'Inquisizione del 1547. Imprigionato nel 1549, dopo un anno e mezzo di carcere, si allontanò da Napoli e si riavvicinò alla Spagna andando al servizio di Cesare d'Avalos, fedele e devoto alla monarchia iberica, con il quale si recò a Barcellona dove rimase per qualche tempo. In ogni caso, la sua condotta politica fu oscillante, tanto che, in seguito ad un'altra congiura contro don Pedro de Toledo ordita dal Sanseverino con l'appoggio silenzioso del cardinale Gian Pietro Carafa (il futuro Paolo IV), nel 1552 Cesare venne nuovamente arrestato e condannato alla prigionia perpetua. Solo per formulare un'ipotesi, allora, dal momento che Carafa fu graziato Filippo II in persona non prima del 1578, la sua presenza nel Tempio per Giovanna d'Aragona si può forse spiegare se si considera che egli stesso, proprio nei primi anni dalla condanna all'ergastolo e soprattutto in seguito alla scomparsa del viceré Toledo, voleva probabilmente cercare favori e amicizia tra gli esponenti dell'aristocrazia ispanica in Italia nella speranza che essi potessero intercedere per lui.

Non è qui nostra intenzione soffermarci ulteriormente sugli altri partecipanti alla sezione in lingua spagnola dell'antologia ruscelliana, confidando che questi pochi esempi siano utili per comprendere come, al di là dell'omaggio di routine, l'elogio della «divina» Giovanna -e potremmo dire, per metonimia, dell'intera «Spagna in Italia»- da parte di questi rimatori fosse tutt'altro che disinteressato, e che anzi rispondesse in pieno alle loro esigenze pubbliche e private. Più interessanti sono invece, sul fronte linguistico, alcune riflessioni che scaturiscono dall'analisi del lessico utilizzato da questi poeti italiani che compongono le proprie liriche in castigliano. Nella propria poetica della lode -anzi, sarebbe il caso di parlare qui di una lingua della lode, al limite di una topica - essi ricorrono con una certa frequenza al termine «primor», lemma tipico della lingua e dell'interaweltanschaaung della cortesanía. Primor, che rimanda alla dimensione dell'«eccelleza» in campo sociale, morale e artistico-culturale ${ }^{19}$, di fatto, non è un vocabolo qualunque nella composizione e declinazione dell'alabanza di un principe o di una nobildonna: nel Libro del Cortegiano (I, xxxiv), infatti, lo stesso Baldassar Castiglione lo annoverava tra quei termini di provenienza spagnola che non solo erano degni di far parte dell'elegante e raffinato lessico della corte, ma che a tutti gli effetti erano già «accettati» nel linguaggio della comunicazione

\footnotetext{
${ }^{19}$ Cfr., ad esempio, Sebastián de Covarrubias, Tesoro de la Lengua Castellana o Española, compuesto por el Licenciado Don Sebastián de Covarrubias Orozco, Capellán de Su Magestad, Mastrescuela y Canónigo de la Santa Yglesia de Cuenca, y Consultor del Santo Oficio de la Inquisición(1611), Madrid, Turner, 1977, p. 882.
} 
rinascimentale tanto nella penisola iberica quanto in quella italian ${ }^{20}$. Nel Tempio la valenza culturale, oltre che semantica, del «primor» emerge in tutte le sue potenzialità in alcuni versi di Francisco Alonso, che non risparmia una certa tensione iperbolica - quasi da mistica neoplatonica - nella lode di Giovanna:

Soys Diosa de hermosura entre las Diosas,

si Diosas puede haver acà entre nos.

Retrato soys d'aquel que en sola vos puso el propio primor de todas cosas.

$(\text { vv. 11-12) })^{21}$

La donna appare come riflesso medesimo del Creatore, che in lei ha posto l'eccellenza in ogni sfera dell'essere. Siamo di fronte a una celebrazione non solo della dama, ma dell'intero codice cortigiano: la perfetta integrazione del primor all'interno del lessico dell'eccellenza testimonia di come il ricorso a tale vocabolo divenga inevitabile dinanzi alle esigenze encomiastiche, e ciò proprio perché esso condensa, a livello semantico, l'idea suprema della dignità nelle diverse occasioni del vivere civile e sociale. Anche Odoardo Gómez, dalle chiare origini spagnole, nel suo sonetto Gran impetu me toma, y gran desseo elogia il «primor», anzi i diversi «primores», di Giovanna d'Aragona sulla stessa falsariga dell'esempio precedente:

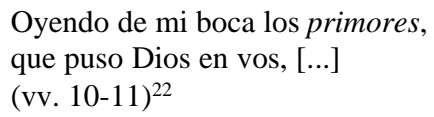

E ancora varia sul tema, e sul vocabolo, Iuan Garçia de Brinag, che in ottave di «arte mayor», seguendo dunque uno degli schemi metrici più consacrati della tradizione iberica pre-petrarchista, estende le lodi riservate alla nobildonna a tutta la sua illustre famiglia, gli Aragona:

\author{
Y pues, quien osara meterse sin guia, \\ por muchos que tenga de ingenio primores \\ a querer contar los predecessores, \\ de donde desciende tu genealogia? \\ Ninguno con seso dezir osaria, \\ en alto linaje ser mas los Troianos, \\ ni aquellos famosos, y grandes Romanos
}

\footnotetext{
20 Per un'analisi dettagliata della rilevanza linguistica e semantica del termine «primor» all'interno della riflessione cortigiana tanto in Italia quanto in Spagna, rimandiamo comunque, direttamente, a un nostro precedente saggio: M. Lefèvre, "Baldassar Castiglione e gli ispanismi nel Cortegiano (I, XXXIV). Note su un episodio di autocoscienza linguistica, culturale e politica", in Philogia Hispalensis, XVIII, 2004, pp. 7-21.

21 F. Alonso, Quien me darà la boz, dama excelente, in Il Tempio alla divina signora donna Giovanna d'Aragona..., cit., p. 156. Corsivo mio. Una certa confusione regna in realtà nella numerazione delle pagine del volume ruscelliano, probabilmente per le progressive inquadernazioni delle parti/sezioni della raccolta. Contiamo qui le pagine, indipendentemente dall'inesatta numerazione della stampa, a partire dalla sezione latina, che a sua volta inizia dopo l'intera serie dei componimenti in italiano.

22 O. Gómez, Gran impetu me toma, y gran desseo, ivi, p. 146. Corsivo mio. Sappiamo poco di questo personaggio, ma è probabile che possa aver avuto qualche importante incarico per conto della Spagna nei territori della Serenissima o comunque che fosse legato in qualche modo al mondo dell'editoria lagunare, dal momento che lo stesso Girolamo Ruscelli gli avrebbe dedicato pochi anni dopo nientemeno che il suo fortunato Rimario, pubblicato sempre a Venezia, presso Giovanni Battista e Melchiorre Sessa, nel 1559.
} 
ser mas poderosos en su monarquia.

$(\text { vv. } 25-32)^{23}$

Qui, in realtà, il termine è utilizzato in senso inverso, per sottolineare più che altro le difficoltà di colui che, per quanto dotato di valore - «primor» appunto -, dovesse cimentarsi nell'impresa di elencare tutti i gloriosi rappresentanti della stirpe aragonese; tuttavia, è significativo notare ancora una volta come questo lemma ritorni insistemente nel lessico e nel linguaggio alto del Rinascimento italospagnolo ad indicare la «cumbre» di qualsiasi attività. Possiamo inoltre congetturare che l'estensione dell'alabanza in onore di Giovanna a tutti gli Aragona, oltre a rientrare agevolmente nella casistica cortigiana, sia dettata da un'origine catalano-aragonese dell'autore, che il nome Garçia de Brinag sembra tradire e che potrebbe suonare altresì come nobile rivendicazione di appartenenza, confermata, forse, anche dalla scelta di un tipo di lirica tradizionale (l'«octava de arte mayor»). E un discorso simile allora si potrebbe fare anche per Hernando Afrodiseo d'Aragón, il cui appellativo non sembra lasciare dubbi in fatto di natali, il quale indugia sul «primor» della dedicataria fin dalla prima ottava del suo componimento, redatto anch'esso in «arte mayor»:

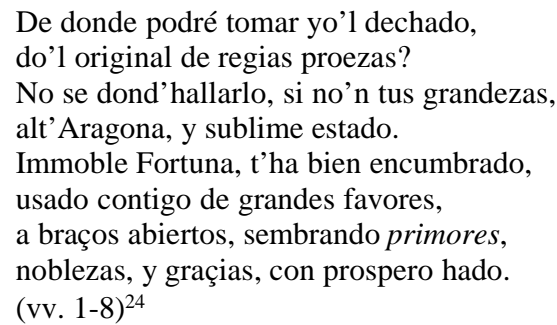

In analogia con l'azione della «Fortuna» nei confronti di Giovanna, anche l'autore a partire da questi versi dissemina il suo testo dei «primores» della nobildonna, che vengono elencati e osannati per tutta la durata della lirica, come mostrano ad esempio le parole usate dal poeta nei versi conclusivi della terza e ultima stanza del suo encomio:

Que tus resplandores tan rezio hirieron mis ojos, que bien lustrar no pudieron tus graçias, tus dotes, tus altos primores. (vv. 30-32) $)^{25}$

In perfetto stile cortigiano, dunque, ancora una volta, il tributo alla dama allude costantemente alla «perfección» della stessa tanto per la sfera morale che per quella culturale e sociale.

Un'attitudine non dissimile è quella che viene in luce anche nelle pagine delle Rime et versi per Giovanna Castriota Carafa $^{26}$. Questa miscellanea, che dal punto di vista

\footnotetext{
${ }^{23}$ I. Garçia de Brinag, Lo mas que yo puedo señora dezir, ivi, p. 148. Corsivo mio.

${ }^{24}$ H. Afrodiseo d'Aragon, De donde podré tomar yo'l dechado, ivi, p. 154. Corsivo mio.

${ }^{25} \mathrm{H}$. Afrodiseo d'Aragon, De donde podré tomar yo'l dechado, ivi, p. 155. Corsivo mio.

${ }^{26} \mathrm{Su}$ questa raccolta, cfr., per un primo sguardo, ancora A. QuONDAM, "Dall'abstinendum verbis alla «locuzione artificiosa». Il petrarchismo come sistema della ripetizione", in G. Ferroni- A. Quondam, La «Locuzione Artificiosa». Teoria ed esperienza della lirica a Napoli nell'età del manierismo Roma, Bulzoni, 1973, in particolare pp. 218-219.
} 
cronologico marca uno scarto in avanti notevole rispetto all'antologia di Ruscelli avvicinandosi nettamente alla fine del secolo, per ciò che riguarda invece premesse e finalità, modalità di costruzione e diffusione - ma soprattutto per la compresenza di liriche in castigliano scritte da autori italiani - segue in modo omogeneo l'esempio del Tempio.

Al termine del volume, si trova una «Tavola de gli autori» con l'intento di notare, dei medesimi, «[...] i nomi, le famiglie, \& le patrie, \& alcune lor qualità [...] $]\rangle^{27}$. Su invito dell’editore, o forse dello stesso curatore Scipione de Monti, questo apparato è in pratica destinato alla lode del lignaggio e dell'abilità poetica dei diversi rimatori antologizzati, e talvolta vi si aggiunge anche qualche informazione biobibliografica relativa ai letterati o ai personaggi più famosi. Responsabile della «Tavola» appare un non meglio precisato Giovan Giacomo de Rossi, il quale, tuttavia, giunto al momento di parlare dei poeti che compongono i sonetti in lingua spagnola, spiazza il lettore con una generica "affettazione di incompetenza" e con la spesa di pochissime parole:

Io non ho molta intelligenza della lingua Spagniuola, né ho molta cognitione de $\mathrm{i}$ personaggi; che scrivono in questo libro, \& però non intendo di parlarne. Ma parmi, che Don Diego Osorio possa contendere con qualunque de nostri, \& così ancho Don Diego Roxas, \& che il Duca di Sessa scriva così bene, come il Petrarca fra noi, Et che i nostri Italiani scrivano così bene in Spagnuolo come gli istessi Spagnioli. ${ }^{28}$

E un singolare e paradossale «descuido» sembra contraddistinguere anche il momento in cui Scipione de Monti introduce direttamente, poche pagine prima della chiusura dell'intero volume, questa sezione spagnola dell'antologia. Egli si limita infatti a una presentazione a dir poco sbrigativa:

Le Rime Spagnuole sono poche, perché si sono smarrite, ma la bontà loro compenserà il difetto del numero. ${ }^{29}$

In realtà, non sappiamo in concreto se questa sorprendente premessa alle rime in lingua spagnola fu annotata dal curatore, dall'editore o da un semplice impiegato della tipografia di Giuseppe Cacchi, che diede alla luce il volume -di fatto, lo stesso de Monti era morto nel 1583 e perciò prima dell'effettiva stampa della miscellanea (1585)-; tuttavia, la millantata incompetenza del compilatore della «Tavola»e la fugacità della premessa a questi «sonetos» non devono far pensare che Scipione de Monti non fosse più che esperto della lingua castigliana, dal momento che egli compone proprio in spagnolo la dedicatoria della sezione («Al Ill.mo Sennor Don Antonio Carrafa») ed è presente - con il nome ispanizzato di «Don Scipion de los Montes» - con varie liriche di scambio all'interno delle ventisette composizioni in castigliano che occupano questa parte. Inoltre, la selezione da lui effettuata esibisce non una semplice e generica conoscenza della lingua spagnola, ma più che altro una discreta capacità critica nei confronti del castigliano letterario.

Prima di osservare come anche in questa raccolta ci troviamo, dunque, di fronte a poeti italiani che scelgono di scrivere nell'idioma dei dominatori, a conferma della confidenza di Scipione con le "cose" di Spagna, e soprattutto con la lingua di quella terra, sono utili infine i paratesti che si trovano all'inizio dell'intera raccolta. Nella Vita di Don Scipione dei Monti, che si trova subito dopo la dedicatoria iniziale e che funziona a mo' di monumento

\footnotetext{
${ }^{27}$ Gio. Giacomo de Rossi, Tavola de gli autori, in Rime et versi..., cit., c. 225.

${ }^{28}$ Ivi, c. 236.

${ }^{29}$ Rime et versi..., cit., p. 215.
} 
funebre per il compilatore del volume, morto - come detto - un paio d'anni prima della stampa dell'opera, si ottengono alcune interessanti notizie su quest'ultimo, tra cui ad esempio la non secondaria circostanza che lo vedeva da anni al servizio proprio dei «signori Castrioti». Il legame tra i de Monti e la Spagna risaliva, tra l'altro, per lo meno alla generazione precedente: il padre di Scipione, Giovan Battista, aveva infatti servito il Gran Capitán, don Gonzalo de Córdoba, mentre il figlio «cominciò nei primi suoi anni à servire l'Imperatore Carlo Quinto di felice memoria, \& poi nelle guerre, che ha fatto Philippo invittissimo Re delle Spagne $\rangle^{30}$. E accanto al mestiere delle armi viene ricordata anche la sua formazione culturale: «Egli ha dato sempre opera à gli studij delle belle lettere, dove ha fatto profitto grande, $\&$ ha appreso bene ogni cosa; \& scrive in più lingue, come in lingua Greca anticha \& moderna, in Latina, in Thoscana, in Spagnola, \& Francese, \& intende quasi tutti gli altri linguaggi di Europa $\aleph^{31}$. Nessun dubbio, dunque, al di là dell'elogio di routine, sul fatto che Scipione de Monti, come abbiamo osservato anche per alcuni autori del Tempio, dominasse tanto la lingua spagnola d'uso, che praticava nella gestione dei suoi pubblici uffici, quanto il castigliano letterario, quello dei libri di cavalleria così come quello della tradizione del petrarquismo. Sul fronte dei modelli letterari, e poetici in particolare, quando si pubblicano le Rime et versi la prospettiva è decisamente mutata rispetto agli anni della compilazione della raccolta di Ruscelli: nel caso del Tempio (1555), l'antologia usciva in anni in cui la poesia «a la manera italiana», nell'orizzonte letterario spagnolo, e ovviamente anche in quello della «Spagna in Italia», costituiva ancora un esercizio di pochi, quasi una pratica da iniziati -solo le Obras di Boscán e Garcilaso, di fatto, avevano conosciuto una certa popolarità e il successo editoriale: tra Spagna e Paesi Bassi si potevano già annoverare almeno una decina di ristampe, mentre le due edizioni "italiane", come ricordato, erano state pubblicate a Roma nel 1547 e a Venezia nel 1553-, e perciò i rimatori che si lanciavano nella stesura di sonetti e canzoni in castigliano dovevano avere a disposizione un numero relativamente esiguo di modelli ispanici; la raccolta curata da Scipione de Monti viene invece edita nel 1585 , quindi a ridosso del secolo successivo e soprattutto dopo un trentennio in cui, specie nell'universo iberico, la lirica petrarchista non solo aveva ormai "spopolato" a livello di stile e di gusto, ma aveva altresì raggiunto un alto livello di realizzazione e sperimentazione attraverso autori consacrati sia dall'accettazione in ambito accademico, cioè da parte degli "addetti ai lavori", sia dall'apprezzamento, per certi versi molto più significativo, degli editori e del pubblico. Senza riferire qui sulla circolazione manoscritta di componimenti sparsi e occasionali, pratica per altro in voga durante tutto il secolo, o sui vari canzonieri pubblicati dai petrarchisti iberici della cosiddetta seconda generazione $e^{32}$, il cui rappresentante più maturo e consapevole rimane Fernando de Herrera, entro la data limite del 1585 basti ricordare almeno un autore che godette di chiara fama e discreta distribuzione editoriale sia in Italia che in Spagna, quale Jorge de Montemayor, che senza alcun dubbio fu appunto tra gli esponenti più significativi della poesia italianizante spagnola del secondo Cinquecento e che orientò altresì il gusto di tanti poeti degli ultimi anni del secolo: questi aveva pubblicato, in forma di prosimetro sannazariano, la sua Diana nel 1559 e l'opera aveva presto ottenuto un grande successo in

\footnotetext{
${ }^{30}$ Vita di Don Scipione dei Monti, tratta da gli Elogij di Mons. Paolo Regio Vescovo di Vico Equense ivi, c. 6. ${ }^{31}$ Ibid.

32 Per questa definizione e la distinzione tra «generaciones» di petrarchisti spagnoli, cfr. in particolare J. G. Fucilla, Estudios sobre el petrarquismo en España, Madrid, CSIC, 1960
} 
patria, al punto che anche in Italia venne ristampata più di una volta ${ }^{33}$. Pertanto, sia Scipione de Monti che gli altri poeti che consacrarono in castigliano il proprio omaggio a Giovanna Castriota, rispetto agli autori del Tempio, avevano senz'altro alle spalle un repertorio maggiore di modelli e tipologie di ascendenza petrarquista per quanto riguardava la pratica lirica; e la maggiore esperienza del petrarchismo di questa sezione spagnola, siano i «sonetos» composti da letterati italiani o da membri dell'esercito e dell'aristocrazia iberica in Italia, si riverbera anche nel fatto che in più di una circostanza è possibile riscontrare non (sol)tanto calchi e imitazioni esplicite di autori noti quanto soprattutto - come vedremo in dettaglio - alcuni riferimenti metaletterari tesi alla celebrazione dell'intera tradizione petrarchistica sia italiana che spagnola.

Prima di introdurre alcuni spunti di analisi, riportiamo intanto l'elenco dei sonetti in lingua spagnola contenuti nelle Rime et versi:

\footnotetext{
${ }^{33}$ Basti ricordare qui le due edizioni "italiane" che riprodussero il testo originale dell'opera:Los siete libros dela Diana de Jorge de Monte Mayor .... Milano, per Andrea de Ferrari nel corso di porta Tosa, [circa 1560]; e La Diana de Jorge de Monte Maior, nueuamente corrigida, y reuista por Alonso de Vlloa.Parte primera. Han se anadido en esta ultima impression los verdaderos amores de Abencerrage, y la hermosa Xarifa. La infelice historia de Piramo y Tisbe..., Venezia, por Jo. Comenzini, 1574, edizione che, come si può osservare, raccoglieva insieme al testo della Diana anche altre importanti opere della tradizione novelesca spagnola. A queste due stampe si aggiunga infine - sempre grazie all'accoppiata Ulloa (curatore)-Comenzini (editore) e per giunta nel medesimo anno di quest'ultima - anche la pubblicazione della Segunda parte dell'opera: La Diana de Jorge de Montemaior, compuesta por Alonso Perez ... Parte segunda. Nueuamente corregida y reuista por Alonso Vlloa Venezia, por Io. Comenzini, 1574, originariamente stampata, sulla scia del successo della prima, nel 1564.
} 


\begin{tabular}{|c|c|}
\hline Aníbal Bufalo & En el Reyno de reynos, mas preclaro. \\
\hline César Carafa & Es yà tan grande tu gloriosa fama. \\
\hline Jerónimo Contreras & O tu feliçe siglo alegre canta. \\
\hline Fray Giulio Carafa & Exemplos del passado, y del presente. \\
\hline Alexandre Andrea & Quien dessea ver, y conosçer en tierra («a don Scipion delos Montes»). \\
\hline Scipión de los Montes & Nunca se viò Alexandre unida en tierra («Respuesta de don Scipion») \\
\hline Scipión de los Montes & Pues, que con verso heroyco soberano («A don Alonzo de Erzilla») \\
\hline A. Téllez de Guevara & Si pudiese por arte un templo alçarse («A don Scipion delos Montes») \\
\hline Scipión de los Montes & $O^{\prime}$ si por mi se viesse à Olimpo alzarse («Respuesta de don Scipion») \\
\hline Costantino Ceuli & Desde la sombra, cabe un Ryo sonante («A don Scipion delos Montes») \\
\hline Scipión de los Montes & Bien puedes con tu lira alta y sonante («Respuesta de don Scipion») \\
\hline Scipión de los Montes & Lilio, que blanco lilio de Romanos («A Cesare Lilio») \\
\hline Cesare Lilio & Monti, bien soys la flor de los Romanos («Respuesta del Lilio») \\
\hline Scipión de los Montes & De Sçiençia, y valor, Osorio, osado («A don Diego Osorio») \\
\hline Diego Osorio & Claro Scipion, de l'African translado («Respuesta de don Diego») \\
\hline Scipión de los Montes & Roxas, que de la flor de todas flores («A don Diego de Roxas») \\
\hline Diego de Roxas & $\begin{array}{l}\text { Excelso Monte, en quien de mil colores («Respuesta de d. Diego de } \\
\text { Roxas») }\end{array}$ \\
\hline Scipión de los Montes & Mañanimo Sennor, Cordova invicto («A G. de Cordova Duque de Sesa») \\
\hline Gonzalo de Córdoba & Ben paresçiose à mi invençible Abuelo («Respuesta de Duque») \\
\hline Scipión de los Montes & Pues muerto es Garçilaso, y Luis de Haro («A Ieronimo Contreras») \\
\hline Jerónimo Contreras & Sennor Ylustre, valoroso y raro («Respuesta del Contreras») \\
\hline Iuan Antonio Rossano & Sennor, que con tus ruegos poderosos («a don Scipion delos Montes») \\
\hline Scipión de los Montes & Hercoles, con Atlante poderosos («Respuesta de don Scipion») \\
\hline los Montes & Señor, que con gran fuerça, y firme aliento («a *** don S. delos Montes») \\
\hline Ano & El fiero esfuerço, y el orgulloso aliento («Respuesta a don Scipion») \\
\hline Scip & Señor Iurado, que en çiudad nasçiestes («Al Iurado de Cordova») \\
\hline El Iurado de Cordova & Vos, que de Apolo, y Caliopea nasçistes («Respuesta del Iurado») \\
\hline
\end{tabular}

Come nel caso del Tempio, le biografie di alcuni di questi poeti appaiono piuttosto significative, e tanto più visto che alcuni di essi furono personaggi di spicco della vita politica, militare e letteraria nei domini spagnoli in Italia durante il secondo Cinquecento. Di Giulio Carafa, ad esempio, oltre a rilevare la sua capacità di scrittura in italiano, latino e spagnolo, nella Tavola di de Rossi si segnala principalmente l'attività di traduttore: «Ha tradotto in nostro linguaggio i problemi di Aristotele, e il Galateo del Casa in lingua Castigliana, \& ha scritto le historie delle guerre di Fiandra $\rangle^{34}$. Dell'altro illustre esponente della famiglia Carafa, Cesare, abbiamo già ampiamente parlato a proposito della sua presenza nel volume ruscelliano; qui tuttavia de Rossi, sbilanciandosi rispetto all'iniziale "affettazione di modestia/incompetenza", si perita di osservare che egli «scrive assai bene cosi in lingua Toscana, come Spagnola $\rangle^{35}$, anche se il rilievo ci appare una premura dettata dall'ossequio verso un simile personaggio piuttosto che un'autentica valutazione critica. E ciò sembra confermato dal fatto che, al contrario, Alessandro d'Andrea, che figura con il

${ }^{34}$ Gio. Giacomo de Rossi, Tavola de gli Autori, in Rime et versi..., cit., c. 231. Si ricordi, come abbiamo potuto osservare in precedenza, che è proprio la «Tavola de gli autori» curata da de Rossi a fornire varie utili note biobibliografiche relative ad alcuni di questi poeti. Oltre che alle notizie ivi contenute rimandiamo comunque, anche in questo caso, al Dizionario Biografico degli Italiani, cit.; e alla banca dati della risorsa elettronica EDIT-

16 , cit. Per altro, in realtà, queste ultime due fonti non confermano l'esistenza di una traduzione delGalateo di Della Casa operata e data alle stampe da Giulio Carafa: l'unica versione castigliana del trattato pubblicata in Italia risulta infatti essere quella veneziana (Juan Varisco, 1585) curata da Domingo de Becerra.

${ }^{35}$ Ivi, cc. 226-227. 
nome ispanizzato di «Alexandre Andrea», è "liquidato" come nativo di Barletta e uomo di palazzo e di lettere ${ }^{36}$ e che Cesare Lilio viene a sua volta semplicemente indicato come originario di Presenzano. Di Giovanni Antonio Rossano, napoletano, il curatore afferma invece che «scrive con molta felicità in ogni lingua $\beta^{37}$, elogio che estende poi anche a Costantino Ceuli, di Otranto, che - osserva ancora de Rossi - «scrive in tre lingue, ciò è Latina, Toscana, \& Spagniuola, \& non senza molta vaghezza $)^{38}$. Se comunque i rimatori italiani, a parte i due Carafa e lo stesso Scipione de Monti, non appaiono nomi di primo piano per ciò che concerne la storia e l'attività letteraria cinquecentesca - e ciò che li tiene insieme, oltre alla lode di Giovanna, sembra essere più che altro la comune origine meridionale, e pugliese in particolare (de Monti e Ceuli sono di Otranto, d'Andrea di Barletta ecc.) -, almeno un paio tra i poeti spagnoli qui inclusi appaiono come stelle di prima grandezza nell'universo storico e culturale della Spagna imperiale. Parliamo soprattutto di don Gonzalo de Córdoba, Duca di Sessa, che fu personaggio chiave nella storia delle relazioni letterarie tra l'Italia e la Spagna, visti i legami della famiglia con alcuni dei poeti e degli umanisti più importanti del Viceregno partenopeo, tra cui Luigi Tansillo; del «Iurado de Cordova», che non è altri se non Juan Rufo, uomo esperto di poesia encomiastica dal momento che fu autore, tra le altre cose, dell'Austriada (1584), il grande poema celebrativo dedicato a don Juan de Austria in seguito alle sue grandi vittorie contro i Turchi; e ancora di Jerónimo Contreras, autore qui di due liriche, il quale fu autore, tra le altre cose, della fortunatissima Selva de aventuras (1565), uno degli esempi più significativi della novela bizantina spagnola della seconda metà del secolo ${ }^{39}$.

Le liriche in castigliano delle Rime et versi sono tutte composte in metro italiano -sono tutti sonetti, in particolare-, e perciò, sia che a scriverle siano letterati o uomini d'arme spagnoli, come Diego de Roxas, Alonzo de Erzilla o Alonso Téllez de Guevara, per menzionare alcuni "minori", sia che siano versatili poeti italiani, come appunto il de Monti, i Carafa o Cesare Lilio, esse costituiscono un esempio lampante di petrarchismo spagnolo, o meglio in spagnolo, acquisito e praticato in Italia. Tutti i componimenti in lingua castigliana presenti all'interno di questa miscellanea si inseriscono, infatti, a tutti gli effetti nella logica della comunicazione petrarchistica: al di là delle questioni di carattere tecnico-stilistico e dell'omogeneità linguistica, sono liriche di dedica e di scambio (numerose le «Respuestas» ad un interlocutore precedente); hanno un minimo comun denominatore encomiastico (si tratti della duchessa Giovanna Castriota, a cui tutta la raccolta è indirizzata, o degli stessi

\footnotetext{
${ }^{36}$ Alessandro d'Andrea era peraltro una "vecchia conoscenza" di Ruscelli, dal momento che quest'ultimo aveva curato un'edizione del suo Della guerra di campagna di Roma, et del Regno di Napoli, nel pontificato di Paolo IIII ..., tre ragionamenti nuovamente mandati in luce da Girolamo Ruscelli Venezia, Gio. Andrea Valvassori, 1560. De Rossi aggiunge infine che egli «ha tradotto Leone Imperatore dell'arte della guerra». Ivi, c. 225.

${ }^{37}$ Ivi, c. 229.

${ }^{38}$ Ivi, c. 227. L'anno precedente alla stampa dell'antologia per Giovanna Castriota erano state pubblicate anche le Rime dell'eccellente sig. Constantino Ceuli. Della città d'Otranto, Verona-Bologna, Fausto Bonardi, s.a. [ma non prima del 1584].

39 Selua de auenturas compuesta por el capitan Hieronymo de Contreras...: va repartida en siete libros, los quales tratan de unos estremados amores que un Cauallero de Sevilla llamado Luzman, tuvo con una hermosa doncella llamada Arbolea, y las grandes cosas que le sucedieron en diez años que anduvo pelegrinando por el mundo y el fin que tuvieron sus amores, Barcelona, Claudes Bornat, 1565. Ad ogni modo, per un'analisi più approfondita del testo e della generale produzione dell'autore, rimandiamo qui direttamente alla recente edizione moderna curata da Teijeiro Fuentes: J. de Contreras, Selva de aventuras: 1565-1783, edición, estudio, bibliografía y notas a cargo de Miguel Á. Teijeiro Fuentes, Zaragoza, Institución Fernando el Católico, 1991.
} 
rimatori che si omaggiano tra loro); e fanno riferimento a personaggi ed eventi della cronaca o della storia recente (oltre alle famiglie dei Castriota e dei Carafa-quest'ultima direttamente coinvolta nell'impresa- ci sono diversi richiami al casato degli Alba e a quello dei Sessa). Inoltre, in generale, in base a quanto emerge dal Tempio ruscelliano così come dall'antologia per Giovanna Castriota, il petrarchismo iberico in Italia fiorisce, nella seconda metà del Cinquecento, non solo grazie ai poeti di origine spagnola che si richiamano ai grandi autori presi a modello e imitati tanto nella forma -nel lessico, soprattutto- quanto nel contenuto (Garcilaso e Boscán, come ricordavamo, sul fronte patrio, quanto Petrarca e Bembo sul versante italiano), ma anche grazie agli occasionali e devoti rimatori italiani, i quali si cimentano nella lode dei propri signori direttamente in lingua castigliana. E ciò non appare - o per lo meno non vuole apparire - né qualcosa di eccentrico né un deprecabile esempio di affettazione, poiché il tutto rientra in maniera agevole all'interno di un sistema comunicativo sovralinguistico -quello del petrarchismo appunto- in continuo fermento, che non sembra preoccuparsi in primo luogo dell'individualità linguistica dei singoli poeti, ma che vede nella poesia soprattutto una funzione sociale di primo piano, un codice internazionale ed elegante che a sua volta conferisce eccellenza e dignità agli individui che si prestano al «gioco». Sul piano strettamente formale, poi, lo spagnolo di questi rimatori è piuttosto curato e risponde anch'esso appieno alle convenzioni del classicismo: al di là dei calchi veri e propri o dei versi che riecheggiano palesemente quelli dei più famosi poeti spagnoli del Rinascimento, ci troviamo comunque di fronte a una lingua che si adatta perfettamente, attraverso un lessico piano e di stile elevato, attraverso formule consacrate e dotti rimandi, alle strutture metriche e ritmiche della «manera italiana». Senza entrare nel dettaglio dei singoli componimenti, tutti questi autori, italiani o spagnoli che siano, dimostrano infatti di conoscere a dovere il castigliano letterario, così come esso si era arricchito e raffinato durante il XVI secolo grazie ai contatti con l'italiano dei cortigiani e dei petrarchisti, e di saperlo utilizzare in maniera consapevole all'interno delle «regole» della comunicazione poetica. In questo senso, lo scambio di sonetti tra lo stesso Scipione de Monti e Jerónimo Contreras offre in modo evidente parecchie conferme dell'abilità letteraria e linguistica degli autori di questa sezione, e al contempo cita altresì vari precisi riferimenti per la formazione di un canone del petrarchismo cinquecentesco tra Spagna e Italia.

Scipión de los Montes («A Ieronimo Contreras») Jerónimo Contreras («Respuesta de Contreras»)

Pues muerto es Garçilaso, y Luis de Haro, poetas, cavallieros, y soldados, con Boscán de valor enamorados, y el de Mendoça Don Diego tan claro : quien harà contra à Muerte escudo, y amparo a tal Señora con versos limados, por que del Tiempo non sean sepultados tantos loores del olvido al Faro? Loadla vos, sin pluma buen Contrero, tinta, y papel, que en dezir de repente soys de la vuestra España un nuevo Homero. Cantando d'este Sol resplandesçiente, porque sù nombre buele alto, y ligero entre la mas desconosçida gente.
Sennor Ylustre, valoroso, y raro, del nombre de los dos mas esforçados Scipiones, del mundo tan nombrados, a quien tomo por guya, y por reparo: por mas, que my sçientia rompo, y aro, no puedo con mis versos mas limados allegar à los vuestros encumbrados, pues passays el Petrarca, y Salazaro, a' vos toca loar, gran cavalliero a esta de virtudes clara fuente, en dulçe estilo, altisimo, y severo; $y$ hazer à sù memoria un tal presente (pues yo alcançar no puedo lo que quiero) qual nunca ymaginose humana mente. ${ }^{40}$ 
In primis, questi due sonetti, attraverso la relazione che si stabilisce tra di essi, rispecchiano e confermano in pieno una caratteristica peculiare di questa breve sezione castigliana di liriche, e che in generale sottende all'intera raccolta, cioè l'alto valore di reciprocità che alimenta le scritture e le dediche dei diversi poeti (qui, ad esempio, nella «Respuesta» si mantengono le rime del primo). Come si può facilmente osservare da un'analisi retrospettiva, anche solo sommaria, delle composizioni di questa parte, infatti, ben 22 sonetti su 27 rientrano nella logica petrarchista dello scambio, e il curatore dell'opera, de Monti, risulta essere non solo l'interlocutore privilegiato dei vari rimatori in castigliano, ma altresì il poeta più presente, anche numericamente (12 liriche sono sue), all'interno della sezione. In secondo luogo, a fronte della conoscenza e della coscienza profonda che Scipione dovette avere del canone del petrarchismo iberico, di fresca formazione e ancora in divenire, nel giro di pochi versi egli elenca in serie i più celebri poeti spagnoli della prima metà del Cinquecento - Boscán, Garcilaso, Hurtado de Mendoza -, punti di riferimento dell'intera generazione poetica successiva, alcuni rappresentanti della quale parteciparono costantemente al circuito della comunicazione lirica tra i due paesi e di cui è testimonianza la raccolta stessa. Inoltre, sul fronte opposto, anche Contreras si dimostra aggiornato all'interno del sistema letterario del petrarchismo, e come termini di paragone per la lode del destinatario della sua lirica sceglie nientemeno che Petrarca e Sannazaro, e cioè non solo due mostri sacri della poesia internazionale durante tutto il secolo, ma anche i due "nomi grossi" della penetrazione di una lirica «al itálico modo» nella penisola iberica. Insomma, nel momento di formare il proprio canone "in versi", questi due poeti consacrano alcuni autori che all'altezza del secondo Cinquecento sono ormai considerati dei modelli di riferimento non solo letterario ma anche, e soprattutto, linguistico: per limitarci al fronte spagnolo, ad esempio, Boscán richiamava emblematicamente la lingua, oltre che la cultura, cortigiana; mentre Garcilaso, sulla scia del Canzoniere petrarchesco, rappresentava sì il poeta dell'inquietudine e della sofferenza amorosa, ma si era dimostrato anche il più sapiente ed equilibrato artefice di un lessico che si ispirasse direttamente alle atmosfere liriche dei Rerum Vulgarium Fragmenta e che nello stesso tempo si adeguasse in modo perfetto alle strutture della versificazione italiana.

In conclusione, il contesto culturale della «Spagna in Italia» negli ultimi decenni del secolo fa sì che si sviluppi, di fatto, un sistema integrato, a cui collaborano sia la componente italiana che quella spagnola dell'élite sociale e culturale al potere. E il Sistema se, da una parte, sul piano politico e militare, si fortifica attraverso la costruzione di castelli ed avamposti difesi dalle truppe di Sua Maestà, dall'altra, su quello letterario, e principalmente su quello linguistico, cresce e dà i migliori frutti grazie all'ormai consolidata interazione tra $\mathrm{i}$ letterati delle due nazioni, interazione che funziona all'interno dell'orizzonte del petrarchismo grazie a una serie di norme precise e rigorose, che testimoniano però anche di una cultura viva e aperta: in nome del Maestro, di Petrarca, sia che il canto si eserciti nella lode di una nobildonna sia che consista nella confessione di urgenze pubbliche o private, ciò che conta è l'alta dignità umana della poesia, indipendentemente dall'opzione linguistica. Nell'universo della comunicazione lirica che si definisce in Italia nel secondo Cinquecento i poeti (italiani e spagnoli) - sia che si

\footnotetext{
${ }^{40}$ Scipión de los Montes, Pues muerto es Garçilaso, y Luis de Haro; e J. Contreras, Sennor Ylustre, valoroso, y raro, in Rime et versi..., cit., pp. 218-219. Corsivi miei. Jerónimo Contreras fu letterato abbastanza noto nel secondo Cinquecento e fu autore della Selva de aventuras (Barcelona, 1565), novela bizantina molto letta e più volte ristampata nei decenni seguiti alla princeps.
} 
esprimano nella propria madrelingua sia che, per tutta la serie di ragioni fin qui analizzate, prediligano la "lingua seconda" - sembrano credere soprattutto nei valori del petrarchismo, nelle immagini e nel lessico che li alimenta e sostiene, piuttosto che in una supposta limpieza de idioma. Certo, come dimostrano le due miscellanee esaminate, la scelta del castigliano da parte degli autori italiani si può spiegare facilmente con argomenti legati per lo più alla prospettiva encomiastica e diplomatica, confortata per giunta dalla circostanza di scrivere nella lingua dell'Impero; tuttavia questi poeti, seppure inconsapevoli, forniscono altresì la prova inconfutabile della presenza, negli stati italiani sottomessi alla Spagna, di un orizzonte culturale, prima ancora che linguistico, di grande vivacità: italiani e spagnoli non frequentavano solo le medesime corti, le strade delle città e, probabilmente, anche le medesime file degli eserciti del re, ma condividevano altresì emozioni e attitudini, immagini e parole, a prescindere dalla lingua e dalla cultura di appartenenza, comunicando sullo stesso piano e con pari dignità sotto gli occhi vigili dei numi letterari di ogni popolo Petrarca, Bembo, Garcilaso, Boscán - in nome del rispetto e dell'ammirazione reciproca. Sebbene, come sempre, anche nei decenni finali del secolo è possibile reperire ancora alcuni strascichi delle polemiche primocinquecentesche sul primato delle lingue- dal dibattito tra il latino e i volgari nazionali alle più spregiudicate e "militanti" grammatiche tese a consegnare palme e onori a una lingua rispetto alle altre (si pensi, ad esempio, alle idee formulate da Nebrija a fine Quattrocento) - quello che sembra predominare nella realtà letteraria e linguistica spagnola in Italia alla fine del secolo è piuttosto un principio di tolleranza: entrambe le lingue - italiano e castigliano - appaiono godere di pari diritti e cittadinanza nei domini imperiali così come all'interno del sistema della comunicazione petrarchistica. Il codice del petrarchismo non si lascia infatti rinchiudere in spazi troppo angusti, delimitati dall'esclusività presunta di una singola parlata nazionale, ma, al contrario, elegge a unica discriminante l'utilizzo di un registro stilistico e linguistico alto ed elegante, ereditato dalla tradizione dei Rerum Vulgarium Fragmenta e filtrato attraverso le più interessanti esperienze poetiche cinquecentesche, che funziona indipendentemente dalla lingua scelta dai poeti: tanto nel Tempio quanto nelle Rime et versi, ad esempio, accanto all'italiano e al castigliano compaiono anche liriche in olandese, in latino, in tedesco, che rispondono comunque appieno alle logiche di queste raccolte e che obbediscono anche, e soprattutto, alle «regole» e alle consuetudini del più generale orizzonte del petrarchismo. A partire dalla seconda metà del XVI secolo, è il petrarchismo stesso ad essere divenuto una lingua franca, una sorta di esperanto ante litteram che si sostituisce al latino nelle occasioni più nobili della vita sociale e letteraria; e allora la poesia che si sviluppa in questi anni tra l'Italia, la Spagna e i "quattro angoli" dell'Impero non solo ne rappresenta a tutti gli effetti una elegante sublimazione, che si esercita tanto nella direzione dell'encomio pubblico quanto in quella della confessione intima e privata, ma costituisce anche, forse inconsapevolmente, una prova della speranza di molti cittadini e cortigiani del Vecchio Continente nella possibilità di una ricomposizione, almeno a livello culturale, delle relazioni problematiche tra principi e signori e dei conflitti politici e diplomatici tra i diversi stati, che tuttavia il più delle volte terminarono invece sui campi di battaglia di mezza Europa. 